\title{
Identification of reliable biomarkers of human papillomavirus 16 methylation in cervical lesions based on integration status using high-resolution melting analysis
}

Lu Liu $^{1 \dagger}$, Chunmei Ying ${ }^{1 *+}$, Zhen Zhao ${ }^{2 \dagger}$, Long Sui ${ }^{3^{*+}}$, Xinyan Zhang ${ }^{4}$, Chunyan Qian ${ }^{5}$, Qing Wang ${ }^{3}$, Limei Chen ${ }^{3}$, Qisang $\mathrm{GuO}^{3}$ and Jiangnan Wu

\begin{abstract}
Background: The dynamic methylation of human papillomavirus (HPV) 16 DNA is thought to be associated with the progression of cervical lesions. Previous studies that did not consider the physical status of HPV 16 may have incorrectly mapped HPV 16 methylomes. In order to identify reliable biomarkers for squamous cervical cancer (SCC), we comprehensively evaluated the methylation of HPV 16 depending on the integration incidence of each sample.

Methods: Based on the integration status of 115 HPV 16-infected patients (50 SCC, 30 high-grade squamous intraepithelial lesion [HSIL], and 35 low-grade squamous intraepithelial lesion [LSIL]) and HPV 16-infected Caski cell lines by PCR detection of integrated papillomavirus sequences, we designed a series of primers that would not be influenced by breakpoints for a high-resolution melting (HRM) PCR method to detect the genome methylation.

Results: A few regions with recurrent interruptions were identified in E1, E2/E4, L1, and L2 despite scattering of breakpoints throughout all eight genes of HPV 16. Frequent integration sites often occurred concomitantly with methylated CpG sites. The HRM PCR method showed 100\% agreement with pyrosequencing when 3\% was set as the cutoff value. A panel of CpG sites such as nt5606, nt5609, nt5615, and nt5378 can be combined in reweighing calculations to distinguish SCC from HSIL and LSIL patients which have high sensitivity and specificity ( $88 \%$ and $92.31 \%$, respectively).
\end{abstract}

Conclusions: Our research shows that combination of CpG sites nt5606, nt5609, nt5615, and nt5378 can be used as potential diagnosis biomarkers for SCC, and the HRM PCR method is suitable for clinical methylation analysis.

Keywords: Squamous cervical cancer, Integration, High-resolution melting analysis, Methylation, Cervical lesion

\section{Background}

High-risk human papillomavirus (HPV) 16 is known to be closely associated with cervical squamous cell carcinoma (SCC) worldwide. Persistent HPV infections are considered to drive progression of cervical neoplasia to invasive cervical cancer [1]. Widespread screening of women with

\footnotetext{
* Correspondence: ycmzh2012@163.com; suilong@fudan.edu.cn

'Equal contributors

'Department of Clinical Laboratory, The Obstetrics and Gynecology Hospital of Fudan University, Fangxie Road No. 419, Huangpu District, Shanghai 200001, China

${ }^{3}$ Medical Center of Diagnosis and Treatment for Cervical Diseases, The Obstetrics and Gynecology Hospital of Fudan University, Fangxie Road No. 419, Huangpu District, Shanghai 200001, China

Full list of author information is available at the end of the article
}

the Papanicolaou test has reduced the incidence and mortality due to cervical cancer in countries where it has been systematically implemented. However, some cases are still missed, and otherwise, repeat colposcopy is invasive and difficult to bear for some women. In 2015, the American Society for Colposcopy and Cervical Pathology (ASCCP) and the Society of Gynecologic Oncologists (SGO) convened to provide interim guidance for primary high-risk HPV screening in which high-risk HPV test can be considered alone as alternative to current cervical cancer screening methods [2]. As HPV testing cannot distinguish a persistent infection from a transient infection, it is difficult to predict the risk of cervical cancer from this test alone. Over the past few years, introduction of the HPV 
vaccine has provided evidence of reduced HPV 16/18 infections in countries where there has been high coverage and those who still have no chance to receive HPV vaccine such as Chinese women are increasingly concerned about highrisk HPV infection [3]. A more accurate and sensitive method than HR-HPV test is demanding that can predict risk of developing into cervical cancer.

DNA methylation is thought to occur early in malignant transformation, and methylation has recently been investigated as a more stable biomarker [4]. HPV 16 has a high GC content with part of non-traditional CpG islands. The LCR and L1 genes of HPV 16 have been the focus of most attention [5, 6]. Hypermethylated CpG sites in E2 binding sites are thought to repress E2 expression, resulting in deregulation of the E6/E7 oncogene which leads to carcinogenesis [7]. Alternatively, methylated $\mathrm{CpG}$ sites in the L1 gene are assumed to serve as a defense mechanism against the host's immune system, helping development of persistent infection [8]. Like many other double-stranded circular DNA viruses, HPV often integrates into the human genome, which has previously been suggested to contribute to disease progression [9]. Kalantari et al.'s laboratory research firstly demonstrated that integration of the viral genome into the host chromosome events leads to an alteration in methylation patterns on the viral genome that is dependent upon the type of integration event in selected cell lines [10]. And increased methylation of human papillomavirus type 16 DNA was proved to correlate with viral integration in Bryant et al.'s few vulvar intraepithelial neoplasia samples and all this addressed the need to combine the two factors in any related research [11]. Those investigations either treat both integration and methylation as independent factor in their research or purely detect the methylation of the whole genome while disregarding any influence of the integration status that may illustrate a disguised map of methylation status of their samples [12, 13]. Furthermore, due to the different sample types and methodology used by these studies, there is no uniformity of the results. The conclusions of some studies are even conflicting [12-15].

Recent high-throughput sequencing of HPV 16 DNA in patients of different cervical lesions, combined with cost-effective target selection system technology, has revealed a greater number of tandem repetitive integration events, altered chromatin, copy number variation, and new integration hot spots, making it more complex to calculate the methylation level of HPV 16 [16]. Integration has long been thought to be associated with persistent infection, and frequent interruptions exist within the human genome. However, evaluating integration is not suitable for clinical application due to the irregular appearance in clinical samples, and the underlying pathogenic mechanism remains unclear [17, 18]. Furthermore, the interplay mechanism between integration and methylation and the extent to which integration can influence methylation detection are rarely reviewed especially in clinical sample.

Further knowledge regarding the methylation of HPV 16 considering integration incidence may assist our understanding about pathogenesis modulated by epigenetic mechanisms. In this study, we established a detection of integrated papillomavirus sequence (DIPS) PCR method to investigate the integration status of SCC, high-grade squamous intraepithelial lesion (HSIL), and low-grade squamous intraepithelial lesion (LSIL) samples. Based on the integration status, we designed a series of primers that would not be influenced by breakpoints in order to establish a high-resolution melting (HRM) method to detect the genome methylation. All results were confirmed by pyrosequencing.

\section{Methods \\ Origin of samples}

A total of 480 patients with HSIL or LSIL for whom Pap smear samples were collected (ThinPrep system, Hologic, Germany) at the Obstetrics and Gynecology Hospital affiliated with Fudan University between October 2015 and April 2016 were enrolled in the study. Pathological diagnosis was made based on colposcopy-guided punch biopsy, and cytological diagnosis was made based on the Bethesda system (TBS; revised in 2001). Patients whose cytological diagnosis was consistent with their pathological diagnosis were included in this study. Eighty SCC tissue samples were collected from the tissue bank of the hospital. All samples were stored at $-80{ }^{\circ} \mathrm{C}$ until use. Caski (ATCC ${ }^{\circ}$ CRL-1550 $0^{\mathrm{mm}}$ ) and HeLa (ATCC ${ }^{\circ} \mathrm{CCL}^{-2^{\mathrm{mm}}}$ ) cell lines were purchased from ATCC. DNA was extracted from the cell lines and clinical samples using Sangon magnetic-bead purification kits (Sangon Biotech, China) which can obtain intact DNA. HPV typing was performed by PCR using universal primers MY09/MY11 and sequence confirmation. A total of 115 specimens were found to be positive for HPV 16 DNA (50 cases of SCC, 30 cases of high-grade squamous intraepithelial lesion [HSIL], and 35 cases of low-grade squamous intraepithelial lesion [LSIL]). The DNA of each sample was divided equally into two EP tubes, one for DIPS PCR and the other for HRM PCR. The study was approved by the institutional ethics committee. All patients signed an informed consent form.

\section{Detection of integrated papillomavirus sequence (DIPS) PCR analysis of HPV 16 integration status Primers and double-strand adapter sequences}

Fifteen pairs of primers were designed using Primer Premier 6 software based on previously reported interrupted sites in the HPV 16 genome (see Table 1). The sequences of the double-strand adapter and procedure 
Table 1 Fifteen pairs of primers were designed using Primer Premier 6 software based on previously reported interrupted sites in the HPV 16 genome

\begin{tabular}{|c|c|c|}
\hline Primer & Sequence $\left(5^{\prime}-3^{\prime}\right)$ & Size $(b p)$ \\
\hline E2BSS & F-ccgaccccttatattatggaatctt & \\
\hline E2BSsa & B-cagatgtctttgcttttcttcaggaca & 1461 \\
\hline E1-1 & F-atatcagatgacgagaacgaaaatga & \\
\hline E1-1a & B-gatacaggtgaagatttggtagattttatagta & 2489 \\
\hline E1-2 & F-gaaacaccatgtagtcagtatagtg & \\
\hline $\mathrm{E} 1-2 \mathrm{a}$ & B-agtgggggtggtagcagtcagtatagtagtggaag & 2126 \\
\hline E1-3 & F-gaattatcacagatggtacaatg & \\
\hline E1-3a & B-cgataatgacatagtagacgatagtga & 1521 \\
\hline E2-1 & F-gtaaaacataaaagtgcaattg & \\
\hline E2-1a & B-acttacatatgatagtgaatgtcaacg & 763 \\
\hline E2-2 & F-agttgttgcacagagactccgt & \\
\hline$E 2-2 a$ & B-agtgctccaatcctcactgcag & 975 \\
\hline E2-3 & F-ctttggtgcccaaggcgacg & \\
\hline E2-3a & B-tatgggtcgcggcggagtggttggec & 2553 \\
\hline E2-4 & F-tgatgtgtatgtagacacagacaaaagcagcggacgta & \\
\hline$E 2-4 a$ & B-ataggcagacacacaaaagcaca & 450 \\
\hline L2-1 & F-tagaattacaaactataacacc & \\
\hline L2-1a & B-tctacatatacaaccacttcccatgc & 779 \\
\hline $\mathrm{L} 2-2$ & F-agtacgcctagaggttaatgctg & \\
\hline $\mathrm{L} 2-2 \mathrm{a}$ & B-ctggcctatgtaaagcaactata & 121 \\
\hline L2-3 & F-ataatgtcaggtggac & \\
\hline L2-3a & B-catgttttataaagttgggtggc & 821 \\
\hline L1-1 & F-tccatagatatacattctct & \\
\hline L1-1a & B-ctgtgtcatccaatttatttaata & 791 \\
\hline L1-2 & F-caagcaattgcctgggatg & \\
\hline $\mathrm{L} 1-2 \mathrm{a}$ & B-ctataagtatcttctagtgtgc & 740 \\
\hline L1-3 & F-gcaaaggatccccatgtaacaatg & \\
\hline L1-3a & B-caggtgattgtccaccattagagttaat & 804 \\
\hline E6-E7 & F-cttatattatggaatctttgc & \\
\hline E6-E7a & B-gtccagatgtctttgcttttc & 1522 \\
\hline
\end{tabular}

Primer sets for DIPS-PCR of HPV16 integration analysis. Primers without subscript are used for the first linear PCR and with a subscript are used for the second exponential PCR

were followed as described by Luft et al. [19] with some modifications. These modifications included only using Sau3AI, and we added the Titanium Taq DNA Polymerase and PrimeSTAR DNA polymerase (Takara, Japan) into the reaction mixture to amplify genomic DNA templates of all sizes.

\section{Linear and exponential PCR}

The first phase of linear touchdown PCR was performed as follows: denaturation at $94{ }^{\circ} \mathrm{C}$ for $5 \mathrm{~min}$, followed by 40 cycles consisting of denaturation at $94{ }^{\circ} \mathrm{C}$ for $30 \mathrm{~s}$, annealing for $45 \mathrm{~s}$ from 62 to $50{ }^{\circ} \mathrm{C}$ (decrease of $0.2{ }^{\circ} \mathrm{C}$ per cycle), and extension at $72{ }^{\circ} \mathrm{C}$ for $45 \mathrm{~s}$, and final extension at $72{ }^{\circ} \mathrm{C}$ for $10 \mathrm{~min}$. The second phase of exponential PCR was carried out using the same conditions described above, except $4 \mu \mathrm{L}$ of adapter-specific AP1 primers and $4 \mu \mathrm{L}$ of virus-specific AP2 primers were added.

\section{Sanger sequencing of the PCR amplicons}

The PCR amplicons were electrophoresed on 1.5\% agarose gels stained with $4 \mathrm{~S}$ green (Sangon, China) and read by transillumination. Caski DNA was used as the control. Any products that were not in expected bands were excised from the gel, extracted using the Tiangen Gel Extraction Kit (Tiangen, China), and sent for direct Sanger sequencing (Invitrogen, Thermo Scientific, America). A few amplicons were subjected to TA cloning when direct sequencing was difficult due to a low concentration. All sequencing data were collected to analyze the integration status of each specimen, achieved using DNAstar software and the NCBI BLAST tool.

\section{High-resolution melting PCR analysis of HPV 16 genome methylation \\ Standardization of high-resolution melting PCR}

The HRM primers were designed with Methyl Primer software to screen for methylation of a total of $61 \mathrm{CpG}$ sites in the HPV genome, based on the results of the integration experiments, in order to avoid breakpoints in each sample (see Table 2). None of the targeted amplicons exceeded $200 \mathrm{bp}$ due to the limitations of this method. Part of the unmethylated and methylated LCR, L1, L2, E1, E2/E4, and E6/E7 genes (see Table 2), including their own $\mathrm{CpG}$ sites, were synthesized by Sangon Company according to the HPV 16 genome downloaded from NCBI (Genbank JQ004098.1) and cloned into plasmids. Six pairs of the constructed plasmids were mixed together in a $0,10,25,50,75$, and $100 \%$ methylated to unmethylated ratio to generate standard HRM curves for quantitative analysis. The DNA concentration of all amplified template sequences was combined to analyze the accuracy of the HRM method. DNA concentration was confirmed using a Nanodrop ND-2000 spectrophotometer (Thermo Scientific, America).

\section{High-resolution melting PCR analysis of clinical samples and cell lines}

Bisulfite conversion of DNA $(0.8 \mu \mathrm{g})$ of the 115 clinical samples and the Caski and HeLa cell lines was performed using the EpiTect Bisulfite Kit (Qiagen, Germany) according to the manufacturer's protocol. All unmethylated cytosines in the genome, excluding 5-methyl-cytosines, were converted to uracil and ultimately to thymine during Taq polymerase amplification. The HeLa cell DNA was used as a negative control. 
Table 2 The HRM primers were designed with Methyl Primer software to screen for methylation of a total of $61 \mathrm{CpG}$ sites in the HPV genome, based on the results of the integration experiments, in order to avoid breakpoints in each sample

\begin{tabular}{|c|c|c|c|}
\hline Primer & Sequence $\left(5^{\prime}-3^{\prime}\right)$ & CpG sites & Size (bp) \\
\hline \multirow[t]{2}{*}{ E2BSs } & F-AATTTATGTATAAAATTAAG & nt31, nt37, nt43, & 106 \\
\hline & R-CCTAAAACATTACAATTCTCTT & nt52, nt58 & \\
\hline \multirow[t]{2}{*}{ E2 } & F-TTGAAATTATTAGGTAGTATTTGGTT & nt3412, nt3415, nt3417, nt3433, nt3461, nt3462 & \\
\hline & R-TAATYGTCTATATTTCTTYGATG & & 90 \\
\hline \multirow[t]{2}{*}{ L1-1 } & F-GTIIITGTAGATTIAGATTAGTITITI & nt7034, nt7091, nt7136, nt7145 & 187 \\
\hline & R-AAACAACACTAATTCAACATACATACAA & & \\
\hline \multirow[t]{2}{*}{$\mathrm{L} 1-2$} & F-TGTTAGAATTATATGGCGATAGTT & nt6367, nt6389, nt6457 & 124 \\
\hline & R-CCTTIAATATATAAATCGTCTAATACATT & & \\
\hline \multirow[t]{2}{*}{ L1-3 } & F-TAATTTAGATATATAGCGGTTGGT & nt5927, nt5963 & 81 \\
\hline & R-CTAATACCCACACCTAATAACTAA & & \\
\hline \multirow[t]{2}{*}{ L1-4 } & F-GAGTATAGGGTTATAATAATGGT & nt6581, nt6650 & 109 \\
\hline & R-AAATAAATATAACAACACATAATAACAT & & \\
\hline \multirow[t]{2}{*}{ L1-5 } & F-TTAAGGAGTATTIACGATATGGG & nt6731, nt6796 & 199 \\
\hline & R-ACAATTACCTAAATTACAAACCTATA & & \\
\hline \multirow[t]{2}{*}{ L1-6 } & F-TTGATATATTATTAATATAATTGATTA & nt5600, nt5606, nt5609, nt5615 & 142 \\
\hline & R-CYTTTACYTCYTTTCYTAACATATA & & \\
\hline \multirow[t]{2}{*}{ L1-7 } & F-TGTAAGTACGGATGAATATGTT & nt5709, nt5726 & 81 \\
\hline & R-AAATATCCAACTACAAATAATCTAAA & & \\
\hline \multirow[t]{2}{*}{ L2-1 } & F-AATAAATTGTTATTATTAAATAATGCGAT & nt4240, nt4249, nt4261, nt4270, nt4277 & 120 \\
\hline & R-ATAATATCAAATAAACATATACCTACCTA & & \\
\hline \multirow[t]{2}{*}{ L2-2 } & F-TTGATATTATATTTAAGGTTGAAGGT & nt4427, nt4437, nt4441 & 125 \\
\hline & R-CCAATACGTCCGCCTATAC & & \\
\hline \multirow[t]{2}{*}{ L2-3 } & F-ATAGTAATTAGTAGTATATTTATATTAGG & nt4894, nt4906, nt4924 & 90 \\
\hline & R-TACAACTTTAACCTATTATATTATACG & & \\
\hline \multirow[t]{2}{*}{ L2-4 } & F-TTAGGCGTATTGGTATTAGGT & nt5128, nt5173, nt5179 & 101 \\
\hline & R-ATCATAATAATAATATACCTTAACACCT & & \\
\hline \multirow[t]{2}{*}{ L2-5 } & F-TTTATAATTYYGGTAGGATTTGTA & nt5378 & 150 \\
\hline & R-CTTAATCAATTATATTAATAAATATATCAA & & \\
\hline \multirow[t]{2}{*}{ E6 } & F-TTGTTAATTAGGTGTATTAATTGTTAA & nt494, nt502, nt506, nt539 & 179 \\
\hline & R-TATATCTCCATACATAATTACAACTAA & & \\
\hline \multirow[t]{2}{*}{ E7 } & F-GATAAGTAGAATCGGATAGAGTT & nt701, nt752, nt757, nt765, nt780, nt789 & 137 \\
\hline & R-CCTAATATACCCATTAACAAATCTT & & \\
\hline \multirow[t]{2}{*}{ Clone-LCR } & F-CCTACTAATTGTGTTGTGGT & & 689 \\
\hline & R-CTCCTGTGGGTCCTGAA & & \\
\hline \multirow[t]{2}{*}{ Clone-E6 } & F-GGAACAACATTAGAACAGCA & & 231 \\
\hline & R-GCAATGTAGGTGTATCTCCA & & \\
\hline \multirow[t]{2}{*}{ Clone-E7 } & F-GCAACCAGAGACAACTGA & & 231 \\
\hline & R-GGGCACACAATTCCTAGT & & \\
\hline \multirow[t]{2}{*}{ Clone-E2 } & F-AGCAACGAAGTATCCTCTC & & 187 \\
\hline & R-TGTCCACTGAGTCTCTGT & & \\
\hline \multirow[t]{2}{*}{ Clone-L2 } & F-CCTCTGCGTTAAGGTGTT & & 1504 \\
\hline & R-GAAGGAGCTTGGTCAGTTAT & & \\
\hline \multirow[t]{2}{*}{ Clone-L1 } & F-TTCCAGGGTCTCCACAATA & & 1693 \\
\hline & R-GCACATACAAGCACATACAA & & \\
\hline
\end{tabular}


PCR amplification of the converted DNA was carried out in a $25-\mu \mathrm{L}$ reaction volume containing $18 \mu \mathrm{L}$ of $2 \times$ EpiTect HRM PCR Master Mix (Qiagen, Germany), $1 \mu \mathrm{L}$ of each primer $(10 \mathrm{pmol} / \mu \mathrm{L}), 2 \mu \mathrm{L}$ RNase-free water, and $3 \mu \mathrm{L}$ of template DNA. PCR amplification and HRM were performed in a Light Cycler 480 (Roche) with the following parameters: denaturation at $94{ }^{\circ} \mathrm{C}$ for $5 \mathrm{~min} ; 45$ cycles of touchdown PCR consisting of denaturation at $94{ }^{\circ} \mathrm{C}$ for $30 \mathrm{~s}$, annealing for $30 \mathrm{~s}$ from 65 to $50{ }^{\circ} \mathrm{C}$ (decrease of $0.2^{\circ} \mathrm{C}$ per cycle), and extension at $72{ }^{\circ} \mathrm{C}$ for $30 \mathrm{~s}$; and final extension at $72{ }^{\circ} \mathrm{C}$ for $10 \mathrm{~min}$. The HRM procedure was as follows: $95^{\circ} \mathrm{C}$ for $1 \mathrm{~min}, 40{ }^{\circ} \mathrm{C}$ for $1 \mathrm{~min}, 65^{\circ} \mathrm{C}$ for $1 \mathrm{~s}$, and then continuous acquisition until $95{ }^{\circ} \mathrm{C}$ in $0.02{ }^{\circ} \mathrm{C}$ increments. Samples were all run in duplicate in parallel with the standards. Genescanning software (Roche) was used to normalize the melt curves, and the temperatureshifted and distinct melting curves of each sample were generated. The results and efficiency of bisulfite conversion were confirmed by pyrosequencing.

\section{Statistical analysis}

We used SPSS 21.0 software for all statistical analyses. Linear regression analysis was used to evaluate the association between the results obtained by HRM and the pyrosequencing method. To analyze differences in the levels of methylated CpG sites among different cervical lesions, the non-parametric Kruskal-Wallis and Mann-Whitney $U$ tests were employed. The receiver operating characteristic (ROC) curves were used to calculate the optimal cutoff value and evaluate the negative predict value (NPV) and positive predict value (PPV) for each of the $\mathrm{CpG}$ sites. Multiple linear regression equation and cross-validation were used to reweigh each $\mathrm{CpG}$ site for clinical diagnostic. Statistical assays were all two-sided, and $P<0.05$ was considered to be statistically significant.

\section{Results}

Analysis of HPV 16 integration in cell lines

Fifteen pairs of primers were used to identify integration in the Caski cell line using DIPS PCR. We successfully detected three types of integration in the Caski cell line, 11q14.1, 2q24.1, and Xq27.3, as previously reported by Meissner [20] (Fig. 1). No amplification was found in the HeLa cell line (data not shown).

\section{Identification of HPV 16 integration in the clinical samples}

Overall, $81.7 \%$ of the 115 clinical samples demonstrated integration. The frequency of integration in SCC, HSIL, and LSIL patients was 94\% (47/50), 86.7\% (26/30), and $60 \%(21 / 35)$, respectively. There was a significant difference between the three groups $(P<0.05$, Fig. 2a). Most of the integration events were located in previously reported hot spots in the human genome (see Table 3)
[21]. However, there were no obvious preferential sites in the human genome among the three groups. Furthermore, disruption of HPV 16 was scattered throughout all genes from LCR to L1, with frequencies of $0.71 \%$ (LCR), 0.71\% (E6/E7), 52\% (E1), 18.6\% (E2), 8.6\% (L2), and $19.3 \%$ (L1) moving clockwise, as shown in Fig. $2 \mathrm{~b}$ $\left(P<0.001, X^{2}=185.83\right)$. The relationship between HPV integration and clinicopathological characteristic of the three groups are see in Table 4. There was no correlation between integration in genes and the grade of cervical lesions. However, we noticed that there were more commonly interrupted regions of the HPV genome, including nt1800-nt2400 and nt2600-nt2900 in E1 (63/73), nt3200-nt3600 in E2/E4 (17/26), nt5300-nt5500 in L2 (19/27), and nt5600-nt5900 in L1 (8/12). In addition, several recurrent disruption sites were found, including nt2735 $(n=6), \operatorname{nt} 3467(n=5), \operatorname{nt} 1899(n=8)$, nt5365 $(n=4)$, and nt5619 $(n=4)$, some of which were very close to the CpG sites, and these will be a focus of future research (Fig. 4). Here, the recurrent interruption sites must contain more than three breakup events and more than five interruptions usually happened in the so-called commonly high interruption region. The promoter gene and oncogene E6/ E7 showed little interference by interruptions (1/140 and 1/ 140 , respectively).

\section{Standards for high-resolution melting PCR}

All dilutions of plasmids were successfully amplified with the corresponding primers. Each of the curves generated from HRM PCR exhibited a single peak with a specific melting temperature. All methylated plasmids could be distinguished from dilutions containing 0-100\% methylated plasmids. The quantitative standard curves for calculating the methylation of different $\mathrm{CpG}$ sites are shown in Additional file 1: Figure S1. Several linear analyses were used to assess the linearity of the methylation test, correlation coefficient, and detection limit (see Additional file 1: Figure S1 and Table 5). No amplification was detected for the HeLa cell DNA (data not shown). There was no nonspecific amplification of standard plasmids, as confirmed by subsequent direct sequencing.

\section{Analysis of HPV 16 genome methylation}

All 115 tissue samples and the Caski cell line were successfully amplified in parallel with five pairs of standard plasmids, among which 44 CpG sites showed no or lower methylation (Additional file 1: Figures S1 and S2). We were unable to calculate the methylation of CpG sites in the E1 gene from nt2655 to nt2754 due to its high degree of interruption using current method. In order to verify the accuracy of the HRM PCR method, all positive samples were also subjected to pyrosequencing to identify the methylated CpG sites (Additional file 1: Figure S2). When the cutoff values of pyrosequencing for detecting 


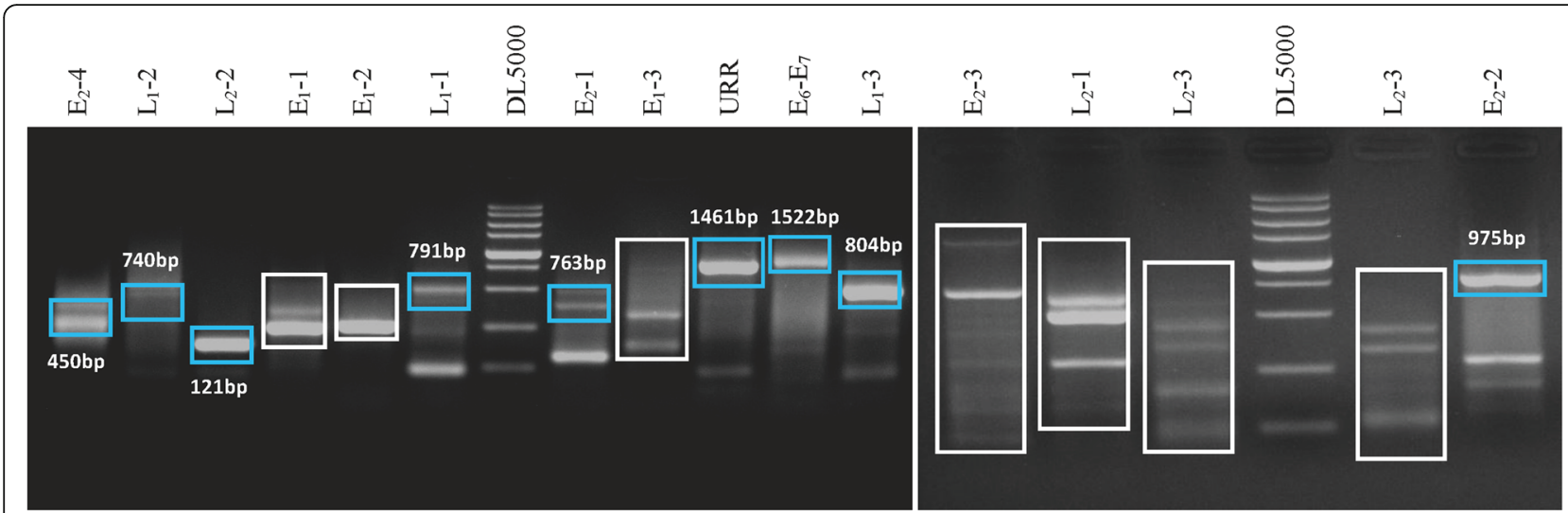

Fig. 1 Confirmation of the primers designed for calculating integration in the Caski cell line. PCR products were electrophoresed on two 1.5\% agarose gels that were run at the same time. The primer used is indicated above the band. DL 5000 was used as the reference marker (Takara, Japan), with bands from the top to the bottom representing 5000, 3000, 2000, 1000, 750, 500, 250, and 100 bp, respectively. DNA bands that were not the expected size are shown in a small box, and these fragments were sent for direct sequencing. There were some recurrent interruptions, and the different band sizes occurred in the same lane

standard plasmids were set as $3 \%$, the two methods showed 100\% agreement (Additional file 1: Figures S1 and S2, Fig. 3). In addition, there were some differences in CpG site location using the HRM method, where different CpG sites in the same CpG island showed differences to the pyrosequencing method (Additional file 1: Figure S2). We found that several CpG sites including nt31, nt37, nt43, nt52, and nt58 were hypermethylated in SCC and HSIL compared to LSIL samples, all of which had a good PPV for distinguishing SCC and HSIL from LSIL (87\%, $86.3 \%, 90.5 \%, 85.7 \%, 84.6 \%$ and $83.1 \%$, respectively; $P<$ 0.05) (Additional file 1: Figure S3). CpG sites nt3412, nt3417, nt3433, nt3436, nt3461, nt3462, nt5600, nt5609, nt5615, and nt5378 showed a high level of methylation in SCC, but a lower level of methylation in HSIL and LSIL which is consistent with Jacquin et al.'s result [7]. Based on the above results, we further reweighed $\mathrm{CpG}$ sites nt5606, nt5609, nt56015, and nt5378 and adjusted the multiple correlation coefficiency (multiple $R=0.84, P<$ 0.001 ) for the relatively small numbers of samples in equations which showed good sensitivity and specificity for distinguishing SCC (see Table 6, $Y>0.5$ ) from HSIL and LSIL $(0<Y<0.5) \quad(88 \%$ and $92.31 \%$, respectively; $P<0$. 001). These panels of $\mathrm{CpG}$ sites are recommended for potential candidate biomarkers combined with other factors for diagnostic test of cervical cancer (Table 6). No defined methylation pattern was found in the three groups, which does not agree with a previous report [22]. Interestingly, we noticed that the high-frequency breakpoints were often accompanied by methylated CpG sites (Fig. 4). A series of CpG sites nt5600, nt5606, nt5609, and nt5615 resides in the commonly interrupted region nt5600-nt5900, and CpG sites nt3462, nt3461, nt3433, nt3417, nt3415, nt3412, and nt5378 are often imbedded in the interruption region nt5300-nt5500 which near the recurrent interrupted sites nt3467 [5] and nt5365 [4] respectively.

\section{Discussion}

A large number of studies have evaluated HPV 16 methylation using traditional methods [23-26]. To the best of our knowledge, we are the first research group to evaluate HPV 16 methylation based on its integration
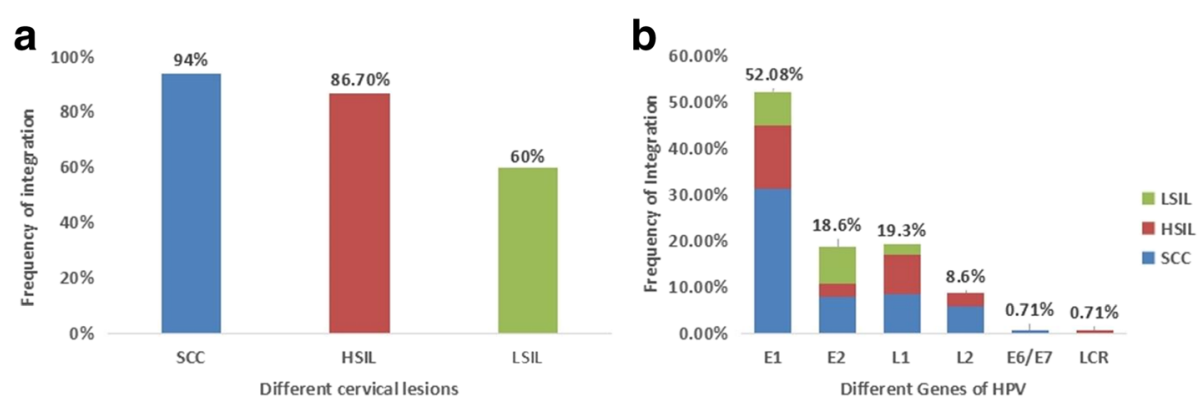

Fig. 2 a Integration frequency of squamous cervical cancer (SCC), high-grade squamous intraepithelial lesion (HSIL), and low-grade squamous intraepithelial lesion (LSIL) $\left(P<0.001, X^{2}=16.605\right)$. b Frequency of integration events of different HPV genes in different groups. All interruptions occurred in these genes, including multiple integration events in the same gene of the same specimen $\left(P<0.001, X^{2}=185.83\right)$ 
Table 3 Most of the integration events were located in previously reported hot spots in the human genome

\begin{tabular}{|c|c|c|c|}
\hline Gene & Locus & Frequency & Virus breakpoint sites \\
\hline MYC & $8 q 24$ & 6 & nt3467, nt3392, nt1886, nt1960, nt2210 \\
\hline SLC7A1 & $1 \mathrm{p} 32.3$ & 2 & nt3467, nt3371 \\
\hline MYF5 & $12 q 21$ & 1 & nt5619 \\
\hline SLITRK5 & $3 q 26.1$ & 2 & nt3867, nt1974 \\
\hline RAB39A & $10 q 26.11$ & 2 & nt5619, nt5311 \\
\hline CD53 & $1 \mathrm{p} 13.3$ & 2 & nt5834, nt2663 \\
\hline TMEM219 & $16 p 11.2$ & 2 & nt5320, nt5365 \\
\hline BRCA1 & $17 q 21$ & 3 & nt3467, nt1869, nt2331 \\
\hline ZFP91 & $19 q 12$ & 5 & nt5812, nt5789, nt1890, nt2759, nt2860 \\
\hline LOC642587 & $1 \mathrm{q} 32.2$ & 1 & nt5365 \\
\hline SLIT3 & $5 q 35.1$ & 1 & nt3272 \\
\hline ZNF595 & $4 \mathrm{p} 16.3$ & 3 & nt5623, nt5521, nt2601 \\
\hline CD34 & $1 q 32.2$ & 2 & nt5619, nt2775 \\
\hline CDK14 & $7 q 21.13$ & 2 & nt5796, nt1903 \\
\hline$R A B 9 B$ & $\mathrm{Xq} 22.2$ & 1 & nt5353 \\
\hline STK24 & $13 q 32$ & 2 & nt2254, nt2684 \\
\hline SH3BP2 & $4 p 16$ & 1 & nt5296 \\
\hline SLITRK2 & $\mathrm{Xq} 27.3$ & 4 & nt5794, nt5321, nt5561, nt2117 \\
\hline TNFAIP2 & $14 q 32.3$ & 2 & nt2735, nt2115 \\
\hline ZNF519 & $18 p 11$ & 2 & nt5826, nt2260 \\
\hline TNFSF15 & $9 q 32$ & 1 & nt5207 \\
\hline SLC4A3 & $2 q 35$ & 2 & nt2789, nt2334 \\
\hline MYOIH & $12 q 24$ & 1 & nt5897 \\
\hline SPANXN1 & $\mathrm{Xq} 27.3$ & 2 & nt2677, nt2892 \\
\hline MRPL17 & 11 p15.4 & 2 & nt1908, nt2741 \\
\hline OR2AG1 & $11 \mathrm{p} 15.4$ & 2 & nt5783, nt2332 \\
\hline FANCC & $9 q 22$ & 2 & nt3467, nt2467 \\
\hline SLCIA3 & $5 p 13.2$ & 1 & nt2887 \\
\hline$\angle R P 1 B$ & $2 q 21$ & 2 & nt5811, nt1899 \\
\hline sox6 & $11 p 15.2$ & 2 & nt2434, nt2561 \\
\hline LOC401097 & $3 q 25$ & 1 & nt3592 \\
\hline SLC13A1 & $7 q 31$ & 1 & nt1899 \\
\hline MYBPC1 & $12 q 23$ & 1 & nt2770 \\
\hline SLC38A4 & $12 q 13$ & 2 & nt1889, nt2654 \\
\hline CYPTA1 & $8 q 12$ & 2 & nt2735, nt1899 \\
\hline ATPBD4 & $15 q 14$ & 2 & nt2017, nt2229 \\
\hline SOX2 & $3 q 26$ & 3 & nt3226, nt2115, nt2095 \\
\hline CADM2 & $3 p 12$ & 3 & nt2735, nt1899, nt2833 \\
\hline LOC728410 & $10 q 26$ & 2 & nt3309, nt3596 \\
\hline PRKX & Xp22 & 3 & nt5964, nt5790 \\
\hline GAP43 & $3 q 13$ & 1 & nt2735 \\
\hline TFPI & $2 q 32$ & 2 & nt3584, nt2607 \\
\hline PPP2R3A & $3 q 22$ & 2 & nt2774, nt2601 \\
\hline MCTP1 & $5 q 15$ & 1 & nt3206 \\
\hline
\end{tabular}


Table 3 Most of the integration events were located in previously reported hot spots in the human genome (Continued)

\begin{tabular}{|c|c|c|c|}
\hline Gene & Locus & Frequency & Virus breakpoint sites \\
\hline$\overline{H L F}$ & $17 q 22$ & 2 & nt5934, nt5821 \\
\hline CLTCL1 & $22 q 11$ & 1 & nt3201 \\
\hline LPPR4 & $1 \mathrm{p} 21$ & 2 & nt5797, nt2735 \\
\hline$S L C 5 A 3$ & $21 q 22$ & 1 & nt1899 \\
\hline ZNF165 & $6 p 22$ & 1 & nt3298 \\
\hline STK24 & $13 q 32.2$ & 1 & nt2392 \\
\hline C5orf39 & $5 p 12$ & 1 & nt1899 \\
\hline PCDH17 & $13 q 21$ & 1 & nt3577 \\
\hline STK11 & 19p13.3 & 1 & nt1923 \\
\hline TMEM26 & $10 q 21$ & 1 & nt3318 \\
\hline$H L A-E$ & $6 p 22$ & 1 & nt5867 \\
\hline FAM82A1 & $2 p 22.2$ & 1 & nt2397 \\
\hline CD44 & $11 p 13$ & 2 & nt3215 \\
\hline FAM131C & $1 p 36.13$ & 1 & nt6854 \\
\hline$S \angle B P$ & $4 \mathrm{p} 16.3$ & 1 & nt2662 \\
\hline SOX17 & $8 q 12.1$ & 1 & $n t 2735$ \\
\hline PRKCA & $17 q 24.2$ & 1 & nt3596 \\
\hline PAH & $12 q 23.2$ & 1 & nt7458 \\
\hline
\end{tabular}

Integration gene and frequency of the human genome and breakpoints of HPV16 occurred in all samples

status. The DIPS PCR approach we used is laborintensive but costs less than next-generation sequencing (NGS) and fulfilled our requirements [27]. We found significantly integration difference among the three groups $(P<0.05)$, of which the higher integration frequency compared to previous study may be due to different DNA qualities, groups of histology, or analysis methods $[28,29]$. As magnetic-bead purification kits are used in our research, it can provide us with intact highquality DNA template which is vital to the whole experiment. Moreover, we added a mix of effective enzymes expert in producing amplicons of different sizes that cannot be found in any other similar assay [30].There were also several recurrent interruptions in the HPV 16 genome which seems to be a series of preferential interruption regions in specific genes. E1 was the gene most likely to be interrupted, and the integration usually appeared between nt1800-nt2400 and nt2600-2900. The E2/E4, L1, and L2 genes showed greater integration in nt3200-nt3600, nt5600-nt5900, and nt5300-nt5500, respectively, of which virus and cellular fusion usually occurred within the 3 '-region of the HPV 16 gene. And those highly interrupted region also occurred in Liu et al.'s investigation who tried to enhance the understanding of the precise location of HPV16 integration sites using capture technology combined with next-generation sequencing [31]. In contrast, the E6/E7 oncogene and the promoter gene were rarely interrupted in our research which is lower compared to Liu et al.'s results. And there are also other interruption sites in Caski cell line except the three detected in our research revealed by NGS method [32]. This may partly ascribed to the sensitivity of the limited restriction enzyme methods we used.

Based on the integration status, we used an HRM PCR method to screen the methylation of integrated HPV 16 genome. This versatile approach can be used to analyze SNP, for human leukocyte antigen (HLA) matching and for species identification which has recently been described for methylation analysis [33-35]. In addition to HRM, several other methods have been used to analyze methylation status, each with their advantages and disadvantages. Methylation-specific PCR (MSP) is a traditional method that requires two pairs of primers to be designed for one target gene. This method is not very sensitive and cannot provide any quantitative information. MethyLight is a real-time method which is more complex and expensive [36]. In contrast, HRM PCR allows for the efficient screening of a sample to rapidly detect the methylation level and is more cost-effective than pyrosequencing which counts every CpG site and requires a specialized instrument [37]. In our research, the HRM method was able to distinguish methylated DNA at a level of $1 \%$ from non-methylated DNA (see Additional file 1: S1, S2 and Table 5). It is a reliable and sensitive method and is more convenient for clinical application when compared with pyrosequencing. All of the expected $\mathrm{CpG}$ sites were successfully detected using carefully designed primers. Some of the CpG sites in L1, 
Table 4 The relationship between clinicopathological characteristic of three groups and HPV integrations

\begin{tabular}{llll}
\hline Variable No. of patients & HPV integrations & P value \\
\cline { 2 - 3 } & Negative & $\frac{\text { Positive }}{n n}$ & No. \% $\%$ \\
\hline
\end{tabular}

SCC patients

Age

$\begin{array}{ll}<40 & 6 \\ 40-50 & 32 \\ >50 & 12\end{array}$

$\begin{array}{llll}0 & 0 & 6 & 100 \\ 2 & 6.25 & 30 & 93.8 \\ 1 & 8.33 & 11 & 91.7\end{array}$

Stage

Stage I 13

Stage II+ 37

Metastasis

Negative 42

Positive 8

Number of pregnancies

$\begin{array}{lllllll}<3 & 21 & 1 & 4.76 & 20 & 95.2 & 0.99 \\ \geq 3 & 25 & 2 & 8 & 23 & 92 & \end{array}$

Number of abortions

$<2 \quad 22$

$\geq 2 \quad 28$

HSIL and LSIL patients

\begin{tabular}{lllllll} 
HSIL & 30 & 4 & 13.3 & 26 & 86.7 & 0.017 \\
LSIL & 35 & 14 & 40 & 0.6 & 60 & \\
Age & & & & & & \\
$\quad<40$ & 52 & 7 & 13.5 & 45 & 86.5 & 0.19 \\
$40-50$ & 11 & 4 & 36.4 & 7 & 63.6 & \\
$>50$ & 2 & 0 & 0 & 2 & 100 & \\
\hline
\end{tabular}

This table shows both the clinicopathological characteristic of SCC, HSIL, and LSIL and their frequency of HPV integrations. $P<0.05$ is considered to be significantly different
Table 5 The fluorescence peak height of a dilution of methylated template in the background of unmethylated template and their correlation coefficiency of different gene standards for methylation analysis of HRM-PCR

\begin{tabular}{|c|c|c|c|c|c|c|c|}
\hline \multirow[t]{3}{*}{ Genes } & \multicolumn{7}{|c|}{ Standards } \\
\hline & \multicolumn{6}{|c|}{ Fluorescence peak height } & \multirow{2}{*}{$\begin{array}{l}\text { Correlation } \\
\text { coefficient }\end{array}$} \\
\hline & $0 \%$ & $1 \%$ & $25 \%$ & $50 \%$ & $75 \%$ & $100 \%$ & \\
\hline$\overline{E S B S S}$ & 69.20 & 51.00 & 42.50 & 33.60 & 21.90 & 5.80 & 0.963 \\
\hline L1-1 & 76.10 & 42.20 & 35.70 & 24.50 & 14.80 & 5.10 & 0.971 \\
\hline L1-2 & 66.20 & 46.70 & 38.20 & 24.00 & 12.30 & 5.80 & 0.983 \\
\hline L1-3 & 56.30 & 48.80 & 40.20 & 28.50 & 12.30 & 3.60 & 0.976 \\
\hline L1-4 & 77.80 & 47.60 & 32.80 & 25.40 & 47.20 & 7.20 & 0.919 \\
\hline L1-5 & 89.40 & 70.90 & 55.00 & 39.00 & 15.00 & 6.90 & 0.953 \\
\hline L1-6 & 66.90 & 49.30 & 32.10 & 25.00 & 12.30 & 2.50 & 0.989 \\
\hline L1-7 & 60.40 & 55.00 & 41.60 & 18.00 & 12.00 & 2.70 & 0.963 \\
\hline L2-1 & 70.30 & 51.90 & 45.00 & 32.50 & 19.30 & 6.10 & 0.967 \\
\hline L2-2 & 69.80 & 44.80 & 32.10 & 22.30 & 10.50 & 1.60 & 0.985 \\
\hline L2-3 & 65.00 & 35.40 & 23.00 & 21.10 & 13.20 & 2.10 & 0.984 \\
\hline L2-4 & 67.50 & 47.70 & 39.00 & 25.90 & 12.30 & 2.10 & 0.959 \\
\hline L2-5 & 70.30 & 41.50 & 30.00 & 21.30 & 15.00 & 2.70 & 0.978 \\
\hline E2 & 66.00 & 55.90 & 42.10 & 23.80 & 12.20 & 2.50 & 0.950 \\
\hline E6 & 68.90 & 47.20 & 35.70 & 30.10 & 24.50 & 7.90 & 0.987 \\
\hline E7 & 66.70 & 55.90 & 48.10 & 26.70 & 25.20 & 5.80 & 0.958 \\
\hline
\end{tabular}

L2, E2, and E2 binding sites showed different methylation levels among the three groups. The E2 binding sites were always hypermethylated in SCC and HSIL and hypomethylated in LSIL, whereas no methylation was detected in the E6/E7 gene in the current study. Epigenetic changes possibly contributed to deregulation of the E2 and E6/E7 gene due to the low frequency of interruption [38]. The non-methylated status of the E6/E7 gene may be partly due to the lack of integration detected by our method [39]. We could not calculate the methylation level of E1 due to the intensive breakpoints within or near the CpG sites; to date, no studies have reported methylation of the
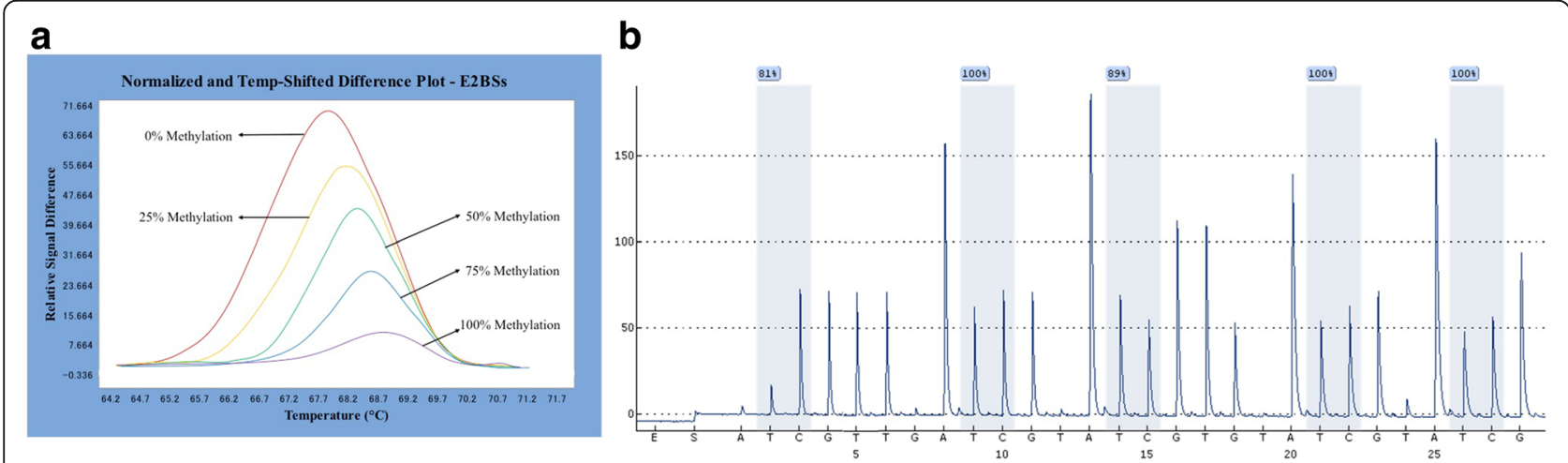

Fig. 3 a Representative HRM standard curves of E2BSs. b The pyrosequencing results of 100\% methylated standard plasmid of E2BSs 
Table 6 Sensitivity and Specificity of the multiple linear regression equation using a panel of CpG sites

\begin{tabular}{llllc}
\hline $\begin{array}{l}\text { Patient numbers of } \\
\text { SCC group }\end{array}$ & $\begin{array}{l}\text { Patient numbers of } \\
\text { predictive SCC group }\end{array}$ & $\begin{array}{l}\text { Patient numbers of predictive } \\
\text { HSIL and LSIL group }\end{array}$ & $\begin{array}{l}\text { Total numbers of each } \\
\text { defined group }\end{array}$ & $\begin{array}{l}\text { Sensitivity\% } \\
(P<0.01)\end{array}$ \\
\hline Patient numbers of SCC group & 44 & 6 & 60 & $\begin{array}{l}\text { Specificity\% } \\
(P<0.01)\end{array}$ \\
Patient numbers of HSIL and LSIL group & 5 & 60 & 65 & 88 \\
\hline
\end{tabular}

The multiple linear regression equation is as follows: $Y=0.006 \mathrm{X} 1+0.15 \mathrm{X} 2+0.29 \mathrm{X} 3+0.93 \mathrm{X} 4-0.008$; multiple correlation coefficiency $R=0.84$, adjusted $R^{2}=0.70, P<0.01 ; \mathrm{X} 1$, $\mathrm{X} 2, \mathrm{X} 3$, and $\mathrm{X} 4$ represent the value of $\mathrm{CpG}$ sites nt5606, nt5609, nt5615, and nt5378, respectively; when $Y>0.5$, this is determined as positive, and when $0<Y<0.5$, this is determined as negative

E1 gene and its interruption may be responsible for neoplasia progression during the virus life circle [40]. We also found limited interruption in E2, and the CpG sites surrounding the frequently interrupted sites were always hypermethylated in SCC. Methylation of E2, which is mainly responsible for E2 repression, showed no interruption effect compared to previous report [41]. We did not detect any methylation of the 3 '-region of the L1-terminus in our sample in contrast with previous inconsistent methylation of the L1 terminus [42, 43]. Hypermethylation of CpG sites in the L1 and L2 genes may be associated with persistent infection by changing the capsid structure, which show no expression in the permissive stage. We further evaluated the most reliable CpG sites nt5606 (NPV $=86.3 \%$, $\mathrm{PPV}=90.6 \%, P<0.001)$, nt5609 $(\mathrm{NPV}=90.9 \%, \mathrm{PPV}=$ $85.9 \%, P<0.001)$, nt5615 (NPV $=86 \%, \mathrm{PPV}=89.2 \%, P<$ $0.001)$, and nt5378 (NPV $=92.8 \%, \mathrm{PPV}=89.1 \%, P<$
0.001) in reweighing calculation with adjusted multiple correlation efficiency using cross-validation for stabilizing multivariable linear regression model and found that the combination of these biomarkers better help us in diagnostic test of SCC (see Table 6). We observed that each sample had its own unique methylation pattern, but were unable to identify any defined pattern that could be used to distinguish the three groups. We speculate that some of the DNA methylome of HPV 16 in human cells may undergo dynamic changes at the same or different stages of cervical disease [44]. In addition, we noticed that the recurrent integration regions were usually accompanied by methylated $\mathrm{CpG}$ sites. In their research, Kalantari et al. observed 3'-region integration of the internal copies of the HPV 16 genome in the concatemer, with hypermethylation of the LCR gene, evaluated using clonal W12 lines derived from the

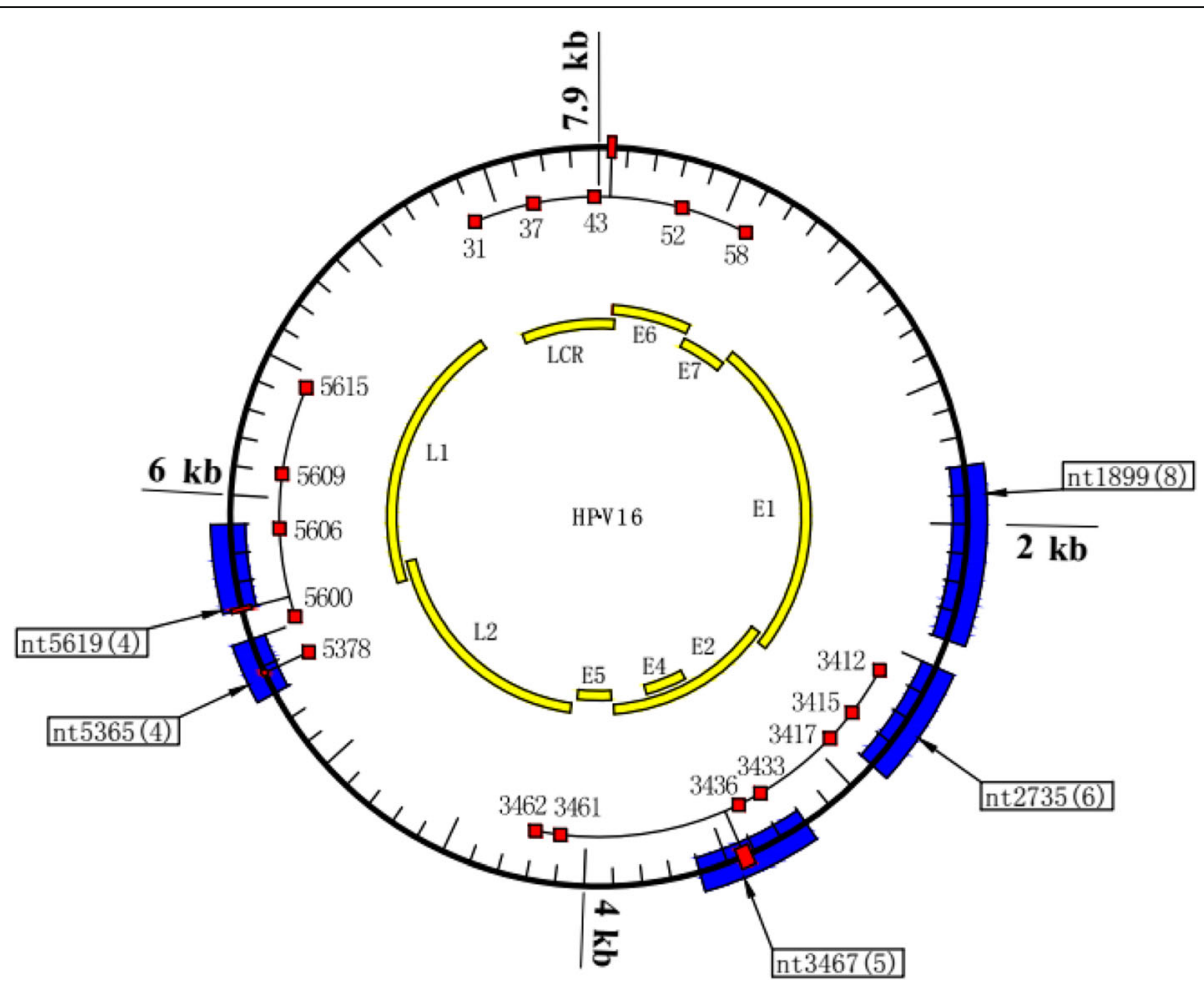

Fig. 4 Recurrent breakpoints and methylated CpG islands near interruption sites are shown in the above map of the HPV 16 genome. The arrows indicate the location and the attached boxes state the number of integrations identified in this study. The red boxes represent the nearby methylated CpG sites. The frequently interrupted regions of E1, E2/E4, L1, and L2 are represented by the blue boxes 
HPV 16-positive cervical intraepithelial neoplasia (CIN) [10]. This supports the hypothesis that inserted copies of the HPV 16 genome are finally silenced by epigenetic changes. Other viruses such as HBV (hepatitis B virus) show that methylation of the $\mathrm{HBx}$ gene is in accordance with the methylation status of the flanking human gene [45]. And also in their HPV research, they showed the DNA methylation status of the integrated HPV16 genome was affected by the methylation status of the human genome flanking integration breakpoints in three neck and head carcinoma cell lines [46].Thus, we suppose that some HPV integrants might be activated or inactivated through self-methylation induced by flanking methylation status of host genome when integrated into human genome, leading to tumorigenesis. Whether this is the case for HPV16induced cervical cancer remains to be confirmed.

For the co-existence of integration and episoma in our samples, the clear relationship between integration and methylation could be further addressed by evaluating mRNA expression or by performing single molecule sequencing. Furthermore, these recommended panel biomarkers in our research need to be assured and optimized the reweighting calculation in the larger multicenter clinical samples. Also, more sensitive method should be used to exhaust all possible integration due to the limitation of DIPS PCR. This research is the first methylation study to consider the genomic state of the virus genome. Comprehensive mapping of the methylomes in HPV 16 based on their integration sites obtained reliable data and will help us to better understand the universal methylation level of HPV 16 or may be the interplay with integration. The research described here highlights the importance of considering the physical status of the virus of which increasingly insert into human genome with the severe disease transformation before methylation detection and promoting new discoveries.

\section{Conclusions}

Our research shows that frequent integration sites often occurred concomitantly with methylated $\mathrm{CpG}$ sites and combination of CpG sites nt5606, nt5609, nt5615, and nt5378 can be used as potential diagnosis biomarkers for SCC, and the HRM PCR method is suitable for clinical methylation analysis.

\section{Additional file}

Additional file 1: The quantitative standard curves for calculating the methylation of different CpG sites. (XLSX $51 \mathrm{~kb}$ )

\section{Abbreviations}

DIPS-PCR: Detection of integrated papillomavirus sequence; HPV: Human papillomavirus; HSIL: High-grade squamous intraepithelial lesion; LSIL: Low-grade squamous intraepithelial lesion; SCC: Squamous cervical cancer

\section{Acknowledgements}

We thank Hongbo Zhao at the Tissue Bank Department of the Obstetrics and Gynecology Hospital of Fudan University for collecting and providing the sample information.

\section{Funding}

This work was supported by the National Natural Scientific Foundation of China (grant nos. 81371874 and 81601831), a grant from the Shanghai Municipal Commission of Health and Family Planning (no. 20154Y0055), and a grant from the Yuhang District Key Scientific Research Project for Medical Sciences (no. 2015008).

\section{Availability of data and materials}

All data generated or analyzed during this study are included in this published article.

\section{Authors' contributions}

The co-authors $L L, C Y, Z Z$, and $L S$ contributed equally to this article in its whole experiment design, clinical recruitment, and statistical analysis. XZ, GQ, $\mathrm{LC}$, and $\mathrm{QW}$ were responsible for collecting all the high-quality samples and their pathological diagnosis. JW also was engaged in the data analysis. All authors read and approved the final manuscript.

\section{Ethics approval and consent to participate}

This research has been approved before it began by the ethics committee of the Obstetrics and Gynecology Hospital of Fudan University. All the patients included in this research had signed informed consent, and no bad effect occurred during this process.

\section{Competing interests}

The authors declare that they have no competing interests.

\section{Publisher's Note}

Springer Nature remains neutral with regard to jurisdictional claims in published maps and institutional affiliations.

\section{Author details}

'Department of Clinical Laboratory, The Obstetrics and Gynecology Hospital of Fudan University, Fangxie Road No. 419, Huangpu District, Shanghai 200001, China. ${ }^{2}$ Department of Clinical Laboratory, Minhang District Central Hospital, Shanghai, China. ${ }^{3}$ Medical Center of Diagnosis and Treatment for Cervical Diseases, The Obstetrics and Gynecology Hospital of Fudan University, Fangxie Road No. 419, Huangpu District, Shanghai 200001, China. ${ }^{4}$ The Research Institute of Obstetrics and Gynecology, The Obstetrics and Gynecology Hospital of Fudan University, Shanghai, China. ${ }^{5}$ Yuhang Branch, Second Affiliated Hospital of Zhejiang University, Hangzhou, China.

${ }^{6}$ Department of Clinical Statistics, The Obstetrics and Gynecology Hospital of Fudan University, Shanghai, China.

Received: 17 September 2017 Accepted: 11 January 2018

Published online: 23 January 2018

\section{References}

1. Moscicki AB, Schiffman M, Kjaer S, et al. Chapter 5: updating the natural history of HPV and anogenital cancer. Vaccine. 2006;24(suppl 3):S42-51.

2. Huh WK, Ault KA, Chelmow D, Davey DD, Goulart RA, Garcia FA, et al. Use of primary high-risk human papillomavirus testing for cervical cancer screening: interim clinical guidance. GynecolOncol. 2015 Feb;136(2):178-82.

3. Harper DM, DeMars LR. HPV vaccines-a review of the first decade. Gynecol Oncol. 2017;146(1):196-204.

4. Clarke MA, Wentzensen N, Mirabello L, Ghosh A, Wacholder S, Harari A, et al. Human papillomavirus DNA methylation as a potential biomarker for cervical cancer. Cancer Epidemiol Biomark Prev. 2012;21:2125-37.

5. Mirabello L, Schiffman M, Ghosh A, Rodriguez AC, Vasiljevic N, Wentzensen $\mathrm{N}$, et al. Elevated methylation of HPV16 DNA is associated with the development of high grade cervical intraepithelial neoplasia. Int J Cancer. 2013;132(6):1412-22

6. Kalantari M, Chase DM, Tewari KS, Bernard HU Recombination of humanpapillomavirus-16 and host DNA in exfoliated cervical cells: a pilot study of L1 gene methylation and chromosomal integration as biomarkers of carcinogenic progression. J Med Virol. 2010;82(2):311-20. 
7. Jacquin E, Baraquin A, Ramanah R, Carcopino X, Morel A, Valmary-Degano S, et al. Methylation of human papillomavirus type $16 \mathrm{CpG}$ sites at E2-binding site 1 (E2BS1), E2BS2, and the Sp1-binding site in cervical cancer samples as determined by high-resolution melting analysis-PCR. J Clin Microbiol. 2013; 51(10):3207-15.

8. Qiu C, Zhi Y, Shen Y, Gong J, Li Y, Li X. High-resolution melting analysis of HPV-16 L1 gene methylation: a promising method for prognosing cervical cancer. ClinBiochem. 2015;48(13-14):855-9.

9. Woodman CB, Collins SI, Young LS. The natural history of cervical HPV infection: unresolved issues. Nat Rev Cancer. 2007;7:11-22.

10. Kalantari M, Lee D, Calleja-Macias IE, Lambert PF, Bernard HU. Effects of cellular differentiation, chromosomal integration and 5-aza-2'-deoxycytidine treatment on human papilloma virus-16 DNA methylation in cultured cell lines. Virology. 2008;374:292-303.

11. Bryant D, Onions T, Raybould R, Jones S, Tristram A, Hibbitts S, et al. Increased methylation of human papillomavirus type 16 DNA correlates with viral integration in vulval intraepithelial neoplasia. J ClinVirol. 2014; 61(3):393-9.

12. Sun $C$, Reimers LL, Burk RD. Methylation of HPV16 genome CpG sites is associated with cervix precancer and cancer. Gynecol Oncol. 2011; 121(1):59-63.

13. Chaiwongkot A, Vinokurova S, Pientong C, Ekalaksananan T, Kongyingyoes $B$, Kleebkaow $P$, et al. Differential methylation of $E 2$ binding sites in episomal and integrated HPV 16 genomes in preinvasive and invasive cervical lesions. Int J Cancer. 2013;132:2087-94.

14. Vinokurova S, von KnebelDoeberitz M. Differential methylation of the HPV16 upstream regulatory region during epithelial differentiation and neoplastic transformation. PLoS One. 2011;6:e24451.

15. Balderas-Loaeza A, Anaya-Saavedra G, Ramirez-Amador VA, Guido-Jimenez MC, Kalantari M, Calleja-Macias IE, et al. Human papillomavirus-16 DNA methylation patterns support a causal association of the virus with oral squamous cell carcinomas. Int J Cancer. 2007;120:2165-9.

16. Hu Z, Zhu D, Wang W, Li W, Jia W, Zeng X, et al. Genome-wide profiling of HPV integration in cervical cancer identifies clustered genomic hot spots and a potential microhomology-mediated integration mechanism. Nat Genet. 2015:47(2):158-63.

17. Wentzensen N, Vinokurova S, von KnebelDoeberitz M. Systematic review of genomic integration sites of human papillomavirus genomes in epithelial dysplasia and invasive cancer of the female lower genital tract. Cancer Res. 2004;64:3878-84

18. Gradíssimo Oliveira A, Delgado C, Verdasca N, Pista A. Prognostic value of human papillomavirus types 16 and 18 DNA physical status in cervical intraepithelial neoplasia. Clin Microbial Infect. 2013;19:E447-50.

19. Luft $F$, Klaes $R$, Nees $M$, Dürst M, Heilmann $V$, Melsheimer $P$, von Knebel Doeberitz M. Detection of integrated papillomavirus sequences by ligationmediated PCR (DIPS-PCR) and molecular characterization in cervical cancer cells. Int J Cancer. 2001;92:9-17.

20. Meissner JD. Nucleotide sequences and further characterization of human papillomavirus present in the CaSki, SiHa and HeLa cervical carcinoma cell lines. J Gen Virol. 1999;80:1725-33.

21. Ziegert C, Wentzensen N, Vinokurova S, Kisseljov F, Einenkel J, Hoeckel M, von KnebelDoeberitz M. A comprehensive analysis of HPV integration loci in anogenital lesions combining transcript and genome-based amplification techniques. Oncogene. 2003;22:3977-84.

22. Patel DA, Rozek LS, Colacino JA, Van Zomeren-Dohm A, Ruffin MT, Unger ER, et al. Patterns of cellular and HPV 16 methylation as biomarkers for cervical neoplasia. J Virol Methods. 2012;184:84-92.

23. Bhattacharjee B, Sengupta S. CpG methylation of HPV 16 LCR at E2 binding site proximal to P97 is associated with cervical cancer in presence of intact E2. Virology. 2006:354:280-5.

24. Wentzensen N, Sun C, Ghosh A, Kinney W, Mirabello L, Wacholder S, et al. Methylation of HPV18, HPV31, and HPV45 genomes and cervical intraepithelial neoplasia grade 3. J Nat Cancer Inst. 2012;104:1738-49.

25. Kalantari M, Garcia-Carranca A, Morales-Vazquez CD, Zuna R, Montiel DP, Calleja-Macias IE, et al. Laser capture microdissection of cervical human papillomavirus infections: copy number of the virus in cancerous and normal tissue and heterogeneous DNA methylation. Virology. 2009:390:261-7.

26. Snellenberg S, Schütze DM, Claassen-Kramer D, Meijer CJ, Snijders PJ, Steenbergen RD. Methylation status of the E2 binding sites of HPV16 in cervical lesions determined with the Luminex $\times$ MAP'M system. Virology. 2012:422:357-65.

27. De Marco L, Gillio-Tos A, Bonello L, Ghisetti V, Ronco G, Merletti F. Detection of human papillomavirus type 16 integration in pre-neoplastic cervical lesions and confirmation by DIPS-PCR and sequencing. J ClinVirol. 2007; 38(7):-13.

28. Bodelon C, Vinokurova S, Sampson JN, den Boon JA, Walker JL, Horswill MA, et al. Chromosomal copy number alterations and HPV integration in cervical precancer and invasive cancer. Carcinogenesis. 2016;37(2):188-96.

29. Olthof NC, Huebbers CU, Kolligs J, Henfling M, Ramaekers FCS, Cornet I, et al. Viral load, gene expression and mapping of viral integration sites in HPV16-associated HNSCC cell lines. Int J Cancer. 2015;136(5):E207-18.

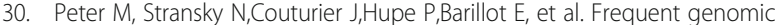
structural alterations at HPV insertion sites in cervical carcinoma. J Pathol. 2010:221:320-30.

31. Liu Y, Lu Z, Xu R, Ke Y. Comprehensive mapping of the human papillomavirus (HPV) DNA integration sites in cervical carcinomas by HPV capture technology. Oncotarget. 2016;7(5):5852-64.

32. Akagi K, Li J, Broutian TR, Padilla-Nash H, Xiao W, et al. Genome-wide analysis of HPV integration in human cancers reveals recurrent, focal genomic instability. Genome Res. 2014;24(2):185-99.

33. Tse MY, Ashbury JE, Zwingerman N, King WD, Taylor SAM, Pang SC. A refined, rapid and reproducible high resolution melt (HRM)-based method suitable for quantification of global LINE-1 repetitive element methylation. BMC Res. 2011:4:6.

34. Wojdacz TK, Dobrovic A. Methylation-sensitive high resolution melting (MS-HRM): a new approach for sensitive and high-throughput assessment of methylation. Nucleic Acids Res. 2007;35:e41.

35. Dimitrakopoulos L, Vorkas PA, Georgoulias V, Lianidou ES. A closed-tube methylation-sensitive high resolution melting assay (MS-HRMA) for the semi-quantitative determination of CST6 promoter methylation in clinical samples. BMC Cancer. 2012;12:486.

36. Weisenberger DJ, Campan M, Long TI, Kim M, Woods C, Fiala E, Ehrlich M, Laird PW. Analysis of repetitive element DNA methylation by MethyLight. Nucleic Acids Res. 2005;33:6823-36.

37. Colella S, Shen L, Baggerly KA, Issa JP, Krahe R. Sensitive and quantitative universal pyrosequencing methylation analysis of $\mathrm{CpG}$ sites. BioTechniques. 2003:35:146-50

38. Piyathilake CJ, Macaluso M, Alvarez RD, Chen M, Badiga S, Edberg JC, Partridge EE, Johanning GL. A higher degree of methylation of the HPV16 E6 gene is associated with a lower likelihood of being diagnosed with cervical intraepithelial neoplasia. Cancer. 2011;117:957-63.

39. Mazumderlndra D, Singh RK, Mitra S, Dutta S, Chakraborty C, Basu PS, et al. Genetic and epigenetic changes of HPV16 in cervical cancer differentially regulate E6/E7 expression and associate with disease progression. GynecolOncol. 2011;123:597-604

40. Kalantari M, Karlsen F, Kristensen G, Holm R, Hagmar B, Johansson B. Disruption of the E1 and E2 reading frames of HPV 16 in cervical carcinoma is associated with poor prognosis. Int J GynecolPathol. 1998; 17(2):146-53.

41. Jeon S, Lambert PF. Integration of human papillomavirus type 16 DNA into the human genome leads to increased stability of E6 and E7 mRNAs: implications for cervical carcinogenesis. Proc Nat AcadSci. 1995;92:1654-8.

42. Bryant D, Tristram A, Liloglou T, Hibbitts S, Fiander A, Powell N. Quantitative measurement of human papillomavirus type 16 L1/L2 DNA methylation correlates with cervical disease grade. J ClinVirol. 2014;59:24-9.

43. Oka N, Kajita M, Nishimura R, Ohbayashi C, Sudo T. L1 gene methylation in high-risk human papillomaviruses for the prognosis of cervical intraepithelial neoplasia. Int J Gynecol Cancer. 2013:23:235-43.

44. Fernandez AF, Rosales C, Lopez-Nieva P, Graña O, Ballestar E, Ropero S, et al. The dynamic DNA methylomes of double-stranded DNA viruses associated with human cancer. Genome Res. 2009;19(3):438-51.

45. Watanabe $Y$, Yamamoto $\mathrm{H}$, Oikawa R, Toyota M, Yamamoto M, Kokudo N, et al. DNA methylation at hepatitis B viral integrants is associated with methylation at flanking human genomic sequences. Genome Res. 2015; 25(3):328-37.

46. Hatano T, Sano D, Takahashi H, Hyakusokul H, Isono Y, Shimada S, et al. Identification of human papillomavirus (HPV) 16 DNA integration and the ensuing patterns of methylation in HPV-associated head and neck squamous cell carcinoma cell lines. Int J Cancer. 2017;140:1571-80. 